IP Periodica Polytechnica Chemical Engineering

61(3), pp. 216-226, 2017

https://doi.org/10.3311/PPch.9504

Creative Commons Attribution (i)

RESEARCH ARTICLE

\section{Modeling of Complex Processes in Turbulent Flow of Unstable Emulsions of Immiscible Liquids}

\author{
Alexander Rozentsvaig ${ }^{1 *}$, Cheslav Strashinskii ${ }^{1}$
}

Received 18 May 2016; accepted after revision 26 September 2016

\begin{abstract}
Turbulent flows of emulsions are associated with the processes of breakage, coalescence and sedimentation of droplets of dispersed liquid. Mechanisms of these physical phenomena that form the equilibrium composition of the droplets of dispersed phase are predetermined by the structure of turbulence. Spectrum of distribution of the dispersed droplets according to the size determines in turn the nature of the interaction with the continuous medium. Therefore, a hydrodynamic model for unstable emulsion (CFD) is completed by discrete population balance model (DPB). It reflects the state of the dispersed phase of the emulsion required to construct an adequate model for CFD. A joint application requires the coordination of the composition and structure of these models for formalizing of the complex interrelationships of physical phenomena in the continuous medium and the dispersed phase of the emulsion.

The key advantages of such specification of the overall structure of the partial models of the CFD consist in that model includes only the mechanisms of breakup, coalescence and sedimentation of the droplets of the dispersed phase, which are really work in the given conditions. Using of a priori theoretical information in the form of mechanisms of basic physical phenomena (MBPP) is proposed, which is necessary for obtaining the desired particular solutions of applied problems on the basis of common CFD and DPB models.
\end{abstract}

\section{Keywords}

CFD flow simulation, population balance model, liquid-liquid emulsions, turbulent flow, model structure, basic physical phenomena

\section{Introduction}

Emulsion of immiscible liquids is widely used in various technological processes and equipment. The state changes and the nature of the interaction between components (phases) of emulsion system determine the effectiveness of various industrial productions. Productivity of technological equipment is connected with the conditions necessary for the completion of the physico-chemical, hydrodynamic and thermodynamic processes in emulsions. However, understanding of their features is hampered by the diversity and ambiguity of the mechanisms of the phenomena that occur while interaction of immiscible fluids.

Modeling of the liquid emulsions behavior, depending on various production conditions, plays an important role in the design, optimization, and operation of production systems. Solving these problems is largely due to the effective use of computer technology and specially configured software (Xing et al. [1], Gao et al. [2]). In this case performance or capability of numerical methods does not always play a crucial role. A more important aspect is the justification of the mathematical models for real conditions in which complex physical phenomena are formed (Ramkrishna [3], Ramkrishna and Mahoney [4]). It is they that provide the quality results of the model numerical research required for the design and management of the production systems.

The modeling complexity of such processes lies in the fact that they do not always appear in full scale. Their composition is formed in a particular way every time, depending on fluid parameters and external conditions. Therefore up to the present there are no universal theoretical concepts, like the classical models for homogeneous liquids. The emulsions heat and substances transfer is controlled largely by the flow regimes, the state and the mechanisms of interaction of the dispersed and continuous phases (Rozentsvaig and Strashinskii [5], Yeoh et al. [6]). For the model calculations in such cases only computational methods of hydrodynamic calculation (CFD) are not enough, the algorithms which are based on the generalization of the concept of continuum mechanics.

In unstable emulsions simulation of heat and substances transfer is associated with the processes of breakage, coalescence and sedimentation of droplets of the dispersed phase, which are of 
a discrete nature. Their model representations are associated by Abidin [7] with the dispersed droplets size distribution. In turn, the range of droplet size determines which of these processes and what mechanisms of these processes can be associated with each of structural modes of turbulent flow of the emulsion. Therefore, the hydrodynamic model of unstable emulsions needs to be complemented by discrete models of population balance (DPB). They detail the composition and relationship of the physical phenomena due to the presence of the dispersed phase (Rosentsvaig and Pergushev [8], Rosentsvaig [9, 10]). However, the formation of droplet size spectrum depends on the flow conditions of emulsion, which are specified by the hydrodynamic model. In turn, the hydrodynamic model of CFD under these conditions should reflect the influence of concentration and non-stationary state of the dispersed phase (Rosentsvaig [11]). Therefore it is necessary to adjust the relationships of the transfer processes on the general phenomenological basis while using both of the two models.

Nature and variety of composition and boundary conditions makes the general mathematical formalization of such processes practically impossible. Without the involvement of the experimental studies data there are problems with the building the phenomenological models of the adequate real physical processes. Insufficient knowledge narrows their model description up to the level of specialized particular presentations. Generalization, extension of their application area requires the use of both theoretical and empirical concepts (Rozentsvaig and Strashinskii [12]).

\section{Composition and structure of DPB model of turbulent flow of unstable emulsions of immiscible liquids}

The general solution to the problem, obtained by a mathematical model in analytical form, represents all possible particular solutions. If the problem solution in an explicit form is absent, so using the specialized software (program code) is necessary, the basis of which are theoretically grounded algorithms. They give the exact particular solutions of mathematically formulated problems under specific initial and boundary conditions. But the question of the adequacy of general models for complex physical ideas require prior, often not less difficult justification. When the software algorithm uses a general method of finding solutions to a mathematical problem, it guarantees the result - the required particular solution. An example is software packages SuperLU aimed at solving problems of linear algebra. A similar situation is in the solution of classical problems of hydrodynamics and heat and mass transfer in fluids by numerical methods of hydrodynamics using CFD software codes type Code_Saturne or PHOENICS.

In contrast to the formal mathematical tasks, for application tasks the question of the correspondence of the model to a real physical problem remains open. The majority of engineering tasks that are of practical interest are associated with the variety of specific working conditions. They are either multi-phase multi-component working fluids, or specialized manufacturing equipment, or the complex physical processes, not only with one of these non-standard conditions, and with different combinations. Therefore, there is the reverse problem of obtaining the required particular solution, it is necessary to specify an appropriate mathematical model.

For definiteness, this article is limited to consideration of horizontal turbulent stream of liquid emulsions. Modellings of transfer processes in heterogeneous systems of immiscible fluids along with the parameters of the continuous media include characteristics of the dispersed phase and the interaction of continuous media and droplets of disperse phases.

Characteristics of the dispersed phase in an unstable emulsion are changed, as is well known, under the influence of turbulent mixing of the continuous medium of such physical phenomena as coalescence, fragmentation and deposition. They determine the structural form of emulsion flow depending on the ratio of the two characteristic dimensions of the dispersed phase: maximum stable with respect to breakup size $d_{\max }$ and the limiting stable size with regard to gravitational deposition $d_{d e p}$.

Fig. 1 represents a diagram of a hierarchical structure where at the first level of discretization models of unstable emulsions are used as the criteria the characteristic droplet size of the dispersed phase $d_{\max }$ and $d_{d e p}$. Under the condition $d_{\max }>d_{d e p}$ the coalescence of droplets can occur up to size when the turbulent flow of emulsion due to the gravitational deposition is transformed into a fully stratified flow. Otherwise, if $d_{\max }<d_{d e p}$ the coalescence does not provide a complete stratification and the flow of the homogeneous fine-disperse or of heterogeneous coarse-disperse emulsion remains.

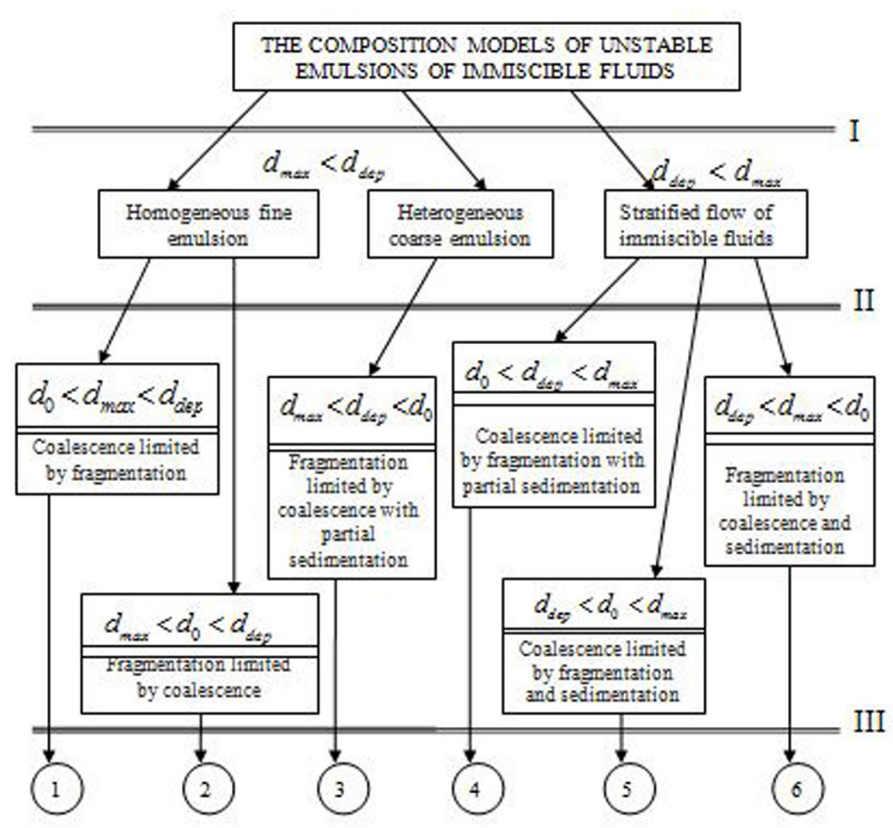

Fig. 1 Modes of turbulent flows of unstable emulsions and the corresponding compositions of the physical phenomena 
Table 1 Schematic images and model representations of MBPP - structural elements of the models

\begin{tabular}{|c|c|c|}
\hline $\begin{array}{l}\text { Mechanisms of basic physical } \\
\text { phenomena }\end{array}$ & Schematic images & $\begin{array}{l}\text { Model representations the basic physeical phenomena } \\
\text { in polydispersed emulsions }\end{array}$ \\
\hline $\begin{array}{l}\text { Model representations the basic physical } \\
\text { phenomena in polydispersed emulsions }\end{array}$ & $\overrightarrow{d_{0}<\lambda_{0}: C G}$ & $\begin{array}{l}\frac{1}{2} \int_{\mathrm{v}_{t_{0}}}^{\mathrm{v}} R_{G}(\mathrm{v}-\mathrm{w}, \mathrm{w}) f_{N}(\mathrm{v}-\mathrm{w}, t) f_{N}(\mathrm{w}, t) d \mathrm{w}- \\
\quad-f_{N}(\mathrm{v}, t) \int_{\mathrm{v}_{t_{0}}}^{\mathrm{v}_{u p}} R_{G}(\mathrm{v}, \mathrm{w}) f_{N}(\mathrm{w}, t) d \mathrm{w} \\
\text { (Rozentsvaig, A., 1988) }\end{array}$ \\
\hline $\begin{array}{l}\text { The pulsation mechanism of droplet } \\
\text { breakup }\end{array}$ & $\overrightarrow{d_{0}<\lambda_{0}: C P}$ & $\begin{array}{l}\frac{1}{2} \int_{\mathrm{v}_{t_{0}}}^{\mathrm{v}} R_{P}(\mathrm{v}-\mathrm{w}, \mathrm{w}) f_{N}(\mathrm{v}-\mathrm{w}, t) f_{N}(\mathrm{w}, t) d \mathrm{w}- \\
\quad-f_{N}(\mathrm{v}, t) \int_{\mathrm{v}_{t_{0}}}^{\mathrm{v}_{u p}} R_{P}(\mathrm{v}, \mathrm{w}) f_{N}(\mathrm{w}, t) d \mathrm{w} \\
\text { (Rozentsvaig, A., 1988) }\end{array}$ \\
\hline $\begin{array}{l}\text { The viscous mechanism of droplet } \\
\text { breakup }\end{array}$ & $\overline{d_{0}}<\overline{\lambda_{0}} \overline{: F} \vec{V}$ & $\begin{array}{l}\int_{\mathrm{v}_{\max , V}}^{\mathrm{up}} \gamma_{V}(\mathrm{w}) g_{V}(\mathrm{v}, \mathrm{w}) f_{N}(\mathrm{w}, t) d \mathrm{w}-\gamma_{V}(\mathrm{v}) f_{N}(\mathrm{v}, t) \\
\text { (Ramkrishna, D., 2000) }\end{array}$ \\
\hline Inertial mechanism of droplet breakup & $\overline{d_{0}}<\overline{\lambda_{0}} \overline{: F} \vec{D}$ & $\begin{array}{l}\int_{\mathrm{v}_{\max , D}} \gamma_{D}(\mathrm{w}) g_{D}(\mathrm{v}, \mathrm{w}) f_{N}(\mathrm{w}, t) d \mathrm{w}-\gamma_{D}(\mathrm{v}) f_{N}(\mathrm{v}, t) \\
\text { (Ramkrishna, D., 2000) }\end{array}$ \\
\hline $\begin{array}{l}\text { Sedimentation of fine droplets in a } \\
\text { turbulent flow of emulsions }\end{array}$ & $\begin{array}{l}\downarrow \downarrow \downarrow \\
d_{0}<\lambda_{0}: S M\end{array}$ & $\begin{array}{l}\int_{\mathrm{v}_{d e p, M}^{\mathrm{v}(H)}}^{\mathrm{v}} \frac{f_{N}(\mathrm{v}) \cdot U_{t u r b}(W, \mathrm{v})}{y} d \mathrm{v} \\
\text { (Rozentsvaig, A., 2003) }\end{array}$ \\
\hline $\begin{array}{l}\text { Sedimentation of coarse droplets in a } \\
\text { turbulent flow of emulsions }\end{array}$ & $\begin{array}{lll}\downarrow & \downarrow & \downarrow \\
d_{0}<\lambda_{0}: S K\end{array}$ & $\begin{array}{l}\int_{\mathrm{v}_{d p p, K}}^{\mathrm{v}(H)} \frac{f_{N}(\mathrm{v}) \cdot U_{t u r b}(W, \mathrm{v})}{y} d \mathrm{v} \\
\text { (Rozentsvaig, A., 2003) }\end{array}$ \\
\hline
\end{tabular}

However, for the transport processes simulation just a detection of the structural form of the flow is not enough. Each structural form corresponds to the set of physical phenomena occurring in the emulsions, which are determined by the current diameter of the droplets of the dispersed phase $d_{0}$. Coalescence in a turbulent flow is possible only in the case when the characteristic size of the droplets of the dispersed phase $d_{0}$ does not exceed the value dmax. In this case coalescing droplets can maintain integrity within the turbulent flow, and the probability of their breakage is negligible. If the size of the droplets $d_{0}$ is greater than $d_{\max }$, it creates favorable conditions for their breakup. The coalescence process under these conditions is limited by the size $d_{\max }$.

The limiting stable size with respect to gravitational deposition $d_{d e p}$ has a similar role in differentiating areas of turbulent flows, where there is a stratification of phases of the emulsion. When the characteristic droplet diameter $d_{0}$ does not exceed $d_{d e p}$, turbulent mixing maintains a uniform quasi-homogeneous flow structure. Otherwise, the energy of turbulent mixing of a continuous medium is not sufficient for a uniform distribution of all the droplets of the dispersed phase in it. Therefore, favorable conditions are created for the partial or complete separation of the turbulent flow.

Hydrodynamic calculations by means of CFD for homogeneous flow are performed on the basis of the finely dispersed emulsion model, like a homogeneous fluid with effective rheology characteristics $\rho_{\mathrm{e}}$ and $\mu_{\mathrm{e}}$. In heterogeneous emulsion flows the interaction of the dispersed phase with the continuous medium is much more complex. To justify the rheological behavior of such heterogeneous systems it is necessary to link the influence of the size and concentration of droplets on the turbulence characteristics. This requires specialized hydrodynamic models and CFD software.

When energy of turbulent mixing is insufficient, the homogeneous emulsion state is broken because of the gravity action. Depending on the range of the droplets sizes, the disperse phase can form simultaneous flow with the emulsion, the concentration in which is reduced up to the complete separation of the emulsion flow. Hydrodynamic calculation of such flows also requires other specialized CFD programs. 
At the third level the turbulence characteristics of the continuous medium of the emulsion are considered within the theory of locally isotropic flows. The micro-scale turbulent fluctuations $l_{0}$ is accepted as a hydrodynamic criterion that allows a differentiating of the transfer processes mechanisms. With the help of a value of microscale in turbulent flows of emulsion two fundamentally different types of interaction of the droplets of the dispersed phase with the continuous medium are highlighted.

The results of the last step of the specification led to the most simple mechanisms of the basic physical phenomena (MBPP). They are a set of elements of the hierarchical structure of the unstable emulsions models, presented in Fig. 2.

For the purpose the current ratio of droplets diameter $d_{0}$ with the microscale of turbulence $\lambda_{0}$ and characteristic diameters of the droplets $d_{\max }$ and $d_{\text {dep }}$ are used. It turned out that on the basis of the accepted criteria generally the implementation of 24 particular structures of the DPB model is possible. For the three selected modes of turbulence of immiscible liquids they consist only of six model elements - (MBPP).

The resulting structure of the physical processes in the emulsions dispersed phase systematizes possible models of their composition and interrelationships on the criteria of the simulation $l_{0}, d_{\max }$ and $d_{d e p}$. Each individual of the MBPP is known and well understood, but it is not enough for specifying realistic mathematical models. The impact of a large number of dimensional variables that are involved in the formation of regularities of real physical phenomena is not always available for theoretical analysis. Lack of a priori theoretical information needs to be filled with the data analysis of experimental research.

A priori empirical information is necessary to ensure the adequacy of model concepts. The hierarchical model of the turbulent flow of unstable emulsions uses it in the form of criteria detail $l_{0}, d_{\max }$ and $d_{d e p}$. Each criterion corresponds to a physical representation, which substantiate empirical model in the generalization of these experimental studies. Note that the criteria themselves represent fairly complex objects of research and their analysis also requires a great attention.

Thus, using the criteria, the full structure of basic mechanisms of physical phenomena in turbulent flows in unstable emulsions is selected. Based on the MBPP the computation DPB-model of adequate complexity and structure for a given specific situation is formulated. In particular, in homogeneous emulsion the DPB-model of CG+CP-FD coalescence type, limited by fragmentation, is written as follows (Rozentsvaig [13]):

$$
\begin{aligned}
\frac{\partial f_{N}(\mathrm{v}, t)}{\partial t}= & \frac{1}{2} \int_{\mathrm{v}_{t_{0}}}^{\mathrm{v}_{\lambda_{0}}} R_{G}(\mathrm{v}-\mathrm{w}, \mathrm{w}) f_{N}(\mathrm{v}-\mathrm{w}, t) f_{N}(\mathrm{w}, t) d \mathrm{w} \\
& +\frac{1}{2} \int_{\mathrm{v}_{\lambda_{0}}}^{\mathrm{v}<\mathrm{v}_{\max , D}} R_{P}(\mathrm{v}-\mathrm{w}, \mathrm{w}) f_{N}(\mathrm{v}-\mathrm{w}, t) f_{N}(\mathrm{w}, t) d \mathrm{w} \\
& -f_{N}(\mathrm{v}, t) \int_{\mathrm{v}_{t_{0}}}^{\mathrm{v}_{\lambda_{0}}} R_{G}(\mathrm{v}, \mathrm{w}) f_{N}(\mathrm{w}, t) d \mathrm{w} \\
& -f_{N}(\mathrm{v}, t) \int_{\mathrm{v}_{\lambda_{0}}}^{\mathrm{v}_{u p}} R_{G}(\mathrm{v}, \mathrm{w}) f_{N}(\mathrm{w}, t) d \mathrm{w} \\
& +\int_{\mathrm{v}_{\max , D}}^{\mathrm{v}_{u p p}} \gamma_{D}(\mathrm{w}) g_{D}(\mathrm{v}, \mathrm{w}) f_{N}(\mathrm{w}, t) d \mathrm{w}-\gamma_{D} f_{N}(\mathrm{v}, t) \\
& \int_{\mathrm{v}_{l_{0}}}^{\mathrm{v}_{u p}} f_{N}(\mathrm{v}, t) d \mathrm{v}=W_{0} \equiv \text { const }
\end{aligned}
$$

where $f_{N}(\mathrm{v}, t)$ - the density distribution of the number of droplets by volume, $W$ - volume concentration of the dispersed phase.

Under conditions of gravitational stratification, the concentration of $W$ and the distribution density of the droplets of the dispersed phase by volume $f_{N}(\mathrm{v}, y, t)$ in polydisperse emulsions change according to the height $y$ of the section $y$ of the tubular device of size $\mathrm{H}$ in accordance with the expressions:

$$
\begin{aligned}
f_{N}(\mathrm{v}, y, t)= & \left.f_{N}(\mathrm{v}, y, t)\right|_{\mathrm{v} \leq \mathrm{v}_{d e p}} \\
+ & \left.f_{N}(\mathrm{v}, y, t)\left(1-\frac{U_{\text {turb }}(W, \mathrm{v}) \cdot t}{y}\right)\right|_{\mathrm{v}_{d e p}<\mathrm{v} \leq \mathrm{v}(H)} \\
W(y, t)=W_{0}- & {\left[\int_{\mathrm{v}_{d e p}}^{\mathrm{v}(H)} \frac{f_{N}(\mathrm{v}, y, t) U_{\text {turb }}(W, \mathrm{v}) \cdot t}{y} d \mathrm{v}\right.} \\
& \left.+\int_{\mathrm{v}(H)}^{\mathrm{v}_{u p}} f_{N}(\mathrm{v}, y, t) d \mathrm{v}\right] .
\end{aligned}
$$

Relationships for the droplets size which are stable with respect to gravitational deposition and for characteristic height $\mathrm{H}$ of the deposition are taken in accordance with the conditions of deposition and with the mechanisms of weighing.

For the model type CG + CP - FD - SK of fragmentation, which is limited by coalescence and with the partial sedimentation the model type CG + CP - FD remains (1). But it is supplemented by relations (3) and (4), in which the general expression of the criterion $\mathrm{v}_{\text {dep }}$ is replaced by specific ratios of volumes $\mathrm{v}_{d e p, M}$ and $\mathrm{v}_{\text {dep, } K}$ for fine and coarse emulsions. 
(3)
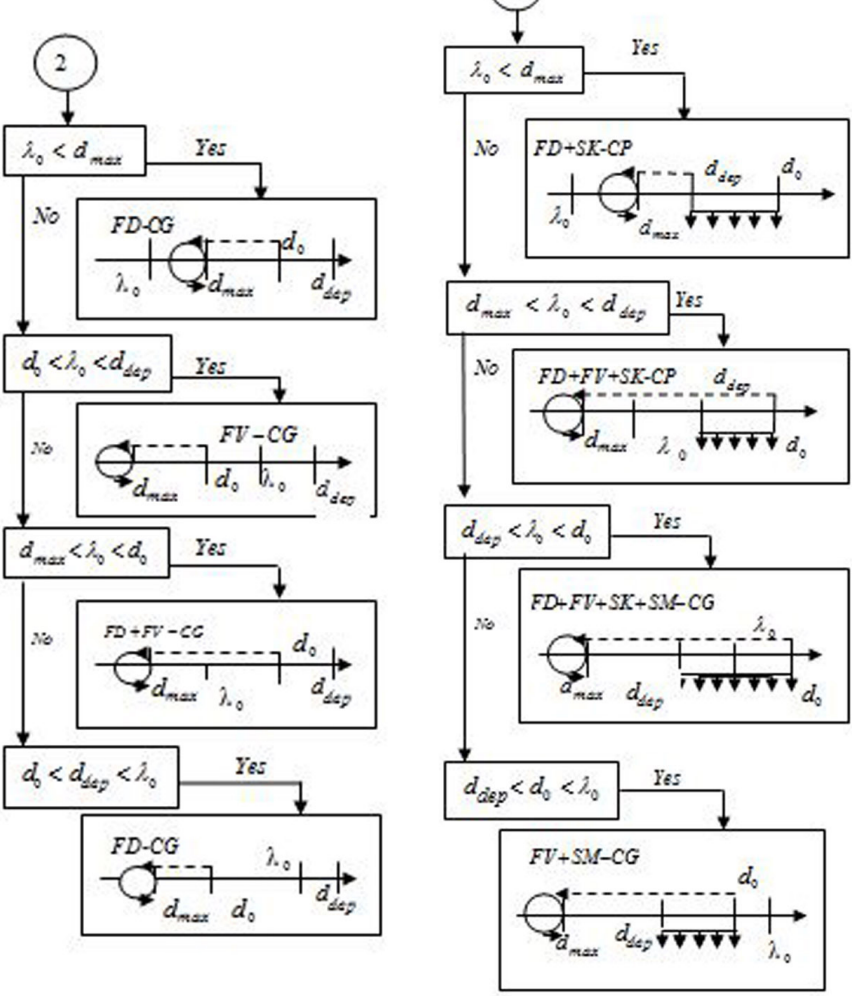

c)

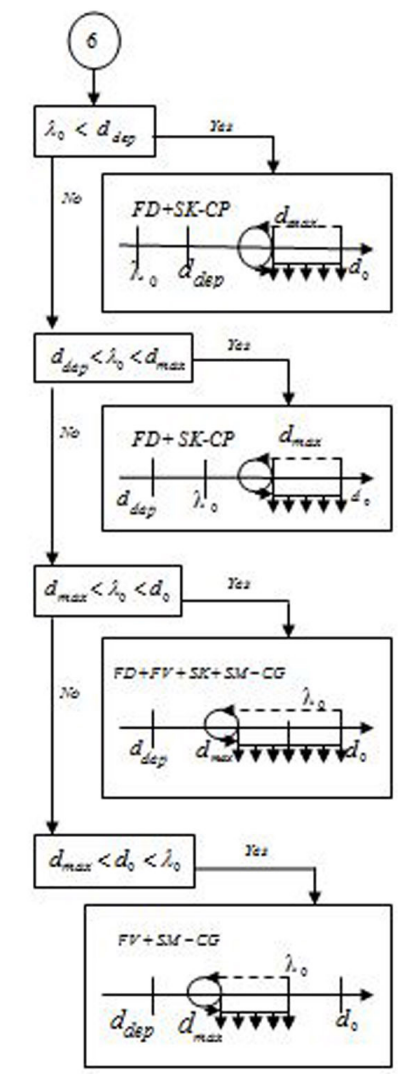

f)

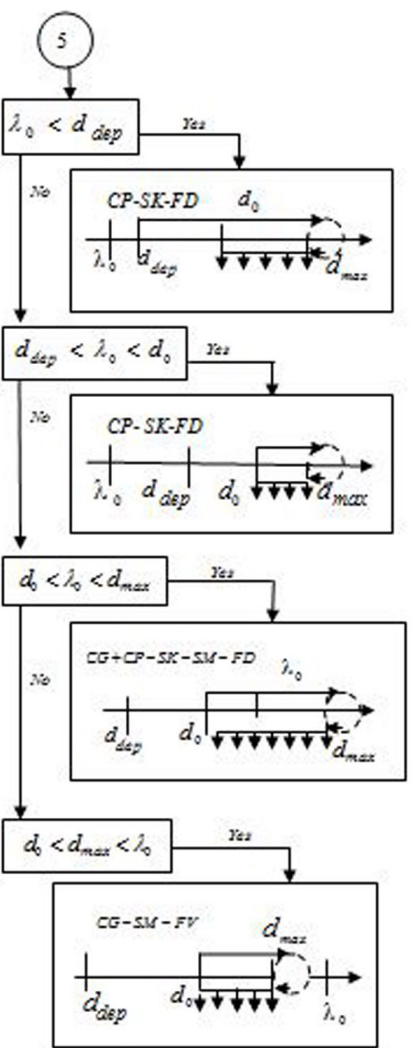

e)
(4)

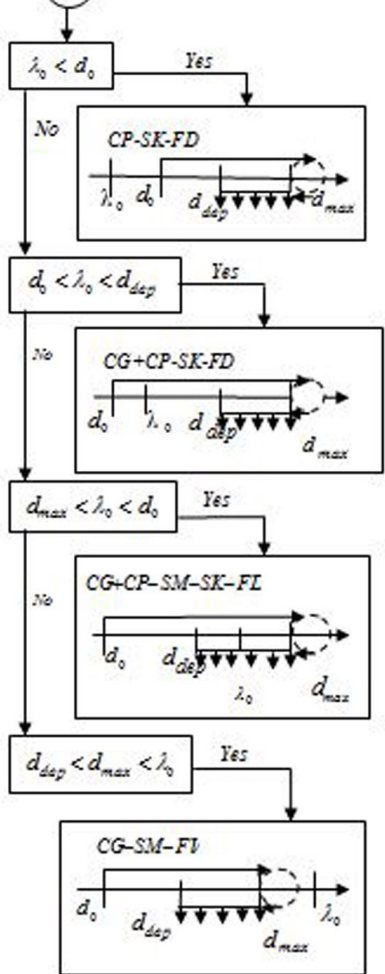

d)

Fig. 2 Schema of possible structures and interrelationships of composition of population balance of the dispersed phase models 


\section{Identification of the interrelated mechanisms of the elementary physical phenomena}

Schematization of complex model representations, represented in Fig. 1 and 2, is largely for demonstration purposes. It illustrates the procedure of building of space (the full composition of elementary physical phenomena) MBPP. Thus composition of full phenomenological model is formalized, related to the features of subject area. When solving practical problems it is usually redundant. To justify the model for the specific terms and conditions in a general form, you need to choose one of 24 possible combinations which are comprised of six structural elements of the MBPP-model.

The MBPP composition is only a necessary condition but it is not enough for an adequate representation the structure of their relationships in the composition of a real physical phenomena. Linear structure, which is often used for the specifications of the CFD and DPB-models, presents a set of competing physical phenomena. For this reason the basic physical phenomena should be statistically independent and should not influence each other. However, the mutual influence is inherent for mechanisms of many physical phenomena. The presence of interaction of such mechanisms leads to non-linear structure of phenomenological models.

Table 2 Structure model representations of complex physical phenomena in emulsions of immiscible liquids.

\begin{tabular}{|c|c|c|}
\hline Model specification & Linear models & Nonlinear models \\
\hline Model elements & Competing additive MBPP & $\begin{array}{l}\text { cross-interaction, } \\
\text { non-additive MBPP }\end{array}$ \\
\hline Nature elements & $\begin{array}{l}\text { Uncorrelated, statistically } \\
\text { independent physical } \\
\text { phenomena }\end{array}$ & $\begin{array}{l}\text { Correlated, } \\
\text { intercorrected } \\
\text { physical phenomena }\end{array}$ \\
\hline $\begin{array}{l}\text { The nature of the } \\
\text { combining elements }\end{array}$ & $\begin{array}{l}\text { Complement or substitute for } \\
\text { each other when participating } \\
\text { in the complex process }\end{array}$ & $\begin{array}{l}\text { Modify each other } \\
\text { and expand the range } \\
\text { of complex process }\end{array}$ \\
\hline
\end{tabular}

The MBPP composition is only a necessary condition but it is not enough for an adequate representation the structure of their relationships in the composition of a real physical phenomena. Linear structure, which is often used for the specifications of the CFD and DPB-models, presents a set of competing physical phenomena. For this reason the basic physical phenomena should be statistically independent and should not influence each other. However, the mutual influence is inherent for mechanisms of many physical phenomena. The presence of interaction of such mechanisms leads to non-linear structure of phenomenological models.

The use of CFD and DPB software can limit the specification of the structure of the physical phenomena in the unstable emulsion that define the particular solution of applied technical problems. Basically, the general theoretical models are usually written in the linear additive form (Zhang at al. [14]). Therefore, the most problematic are the model effects associated with nonlinear nature of the MBPP interaction. They are localized inside of the criteria of detail $d_{\max }, d_{\text {dep }}$ and $l_{0}$, features the model representations of which are described below for turbulent flow of unstable emulsions in pipelines. The subsequent statement does not purport to be a complete analysis. It is presented for the completeness and specificity of argumentation of instructional techniques, which are formalized by the complex model representations.

\subsection{The maximum stable with respect to breakup drop size}

Theoretical analysis of deformation and breakage of the viscous liquid droplets under the influence of dynamic forces and shear stress in an inhomogeneous turbulent flow still remains quite complicated for a complete formalization task. These physical processes are naturally interrelated with hydrodynamic processes of momentum transfer. The nature of such relationships influence not only details of the specifications of agreed CFD and DPB- models, but also conditions that predefine the mechanisms of heat and mass transfer.

In a homogeneous turbulent flow, the droplets breakup of the dispersed phase is associated with velocity fluctuations of the continuous medium of emulsion. The stability of the droplets is provided by surface tension, which resist the external forces of the inertial turbulent fluctuations. Therefore, it is natural to consider the inertial mechanism of droplet breakup as one of basic processes (Kolmogorov [15]). In accordance with the works of Kolmogorov and Hinze the value $d_{\max }$ is determined by the critical value of the Weber number:

$$
W e_{c r i t}=\frac{\rho_{c} \bar{v}^{2} d_{\max }}{\sigma},
$$

where $\sigma$ - surface tension, $\bar{v}^{2}$ - the averaged squared turbulent fluctuations

Another elementary process of droplet breakup by viscous forces in a laminar gradient flow was comprehensively studied by Taylor [16]. Spherical droplets with the diameter $d$ under the influence of shear stress $G$ take the form of an elongated ellipsoid of rotation, close to the cylinder. The magnitude of achievable strain $F$ is represented in the form:

$$
F=\frac{G \cdot d \cdot \mu_{c}}{2 \sigma}\left(\frac{19 \mu_{d} / \mu_{c}+16}{16 \mu_{d} / \mu_{c}+16}\right)
$$

where $\mu_{c}, \mu_{d}$ - viscosity of continuous and dispersed phases of the emulsion

According to Taylor a drop breaks spontaneously into several parts, when the ratio of the longitudinal and transverse dimensions of the cylinder reaches a critical value.

Turbulent flow, when the emulsion moves through the pipelines, in near-wall zone becomes inhomogeneous. 
Consequently, the physical process of breakage also becomes ambiguous. Each of these mechanisms for inhomogeneous turbulent flow is visual, qualitative by nature. You need to validate physical understanding of the nature of the joint occurrence of these two elementary breakup mechanisms.

Only one force of viscous deformation gradient of the averaged velocity is not always enough for a full destabilization the droplets. But the deformed droplet becomes less resistant to the influence of the turbulent fluctuations. Quantitative characterization of these mechanisms for inhomogeneous turbulent flow is possible only on the basis of a priori experimental data.

So well-known feature of droplet breakup for homogeneous turbulence according to Kolmogorov-Hinze is a functional relationship of droplets sizes and average flow rate $d_{\max } \approx \bar{U}^{-1.2}$ . However, as a result of measurements of the diameters of stable droplets in the pipeline a completely different relationship between the maximum stable droplet size and the average flow velocity of turbulent flow was discovered by Slecher [17]:

$$
d_{\max } \approx \bar{U}^{-2.5} \text {. }
$$

Empirical evidence suggests a significant strengthening of influence of the flow velocity in the pipeline. It can be interpreted as the result of the nonlinear interaction of two elementary processes of breakup. It would be incorrect to interpret the result using a simple additive model. A more general procedure for inclusion in inertial mechanism associated deformation gradient droplets is needed (Rozentsvaig [9]).

For this reason, an effective value $d_{e f}$ is added, which is related to the diameter of the original sphere of the same volume with a function of the deformation rate: $d_{e f}=d_{\max } f(F)$. The quantitative effect of droplets deformation of inhomogeneous turbulence (6), which is complementary to relation for the inertial mechanism (5), is estimated using the constant $\alpha$ [9]:

$$
\rho_{c} d_{\max }\left[\frac{\sqrt{\lambda \mu_{c}} \cdot \bar{U}}{\sigma} \varphi\left(\frac{\mu_{d}}{\mu_{c}}\right)\right]^{\alpha} \cdot \frac{\bar{U}^{2}}{\sigma}=C .
$$

To determine the constant $\alpha$ the empirical Sleichercorrelation (7) is used for the flow rate $\alpha+2=2.5$. Hence we obtain a functional expression of the gradient mechanism $d_{e f}=d_{\max } F^{0.5}$ for inclusion in the composition of the physical process through the mechanism of the pulsation breakage.

Thus, the mechanism of destruction of Kolmogorov-Hinze (5) is modified by the inclusion of the gradient mechanism, due to the deformation of the droplets with the gradient of averaged velocity at the wall of the pipeline.

\subsection{The limiting size of droplets of dispersed phase to gravitational deposition}

Modeling of phase stratification of unstable emulsions, when moving in the turbulent regime, also represents a complex physical process (Sarimeseli and Kelbaliyev [18],
Ryazhskikh et al. [19]). Using the scheme in Fig. 2 the particular additive model is dedicated, associated with the sedimentation of dispersed particles in a turbulent flow. For this cross-interaction of basic mechanisms of gravitational settling of droplets and the molecular momentum transfer is represented by the criterion $d_{d e p}$ (Rozentsvaig [20]). But even after the considerable simplifications it is impossible to avoid additional experimental data.

The deposition rate of single spherical droplets of the dispersed phase $V_{d e p}(d)$ relatively to a fixed continuous medium under the action of gravity is determined when $R e_{d}<1$ by the Stokes formula:

$$
V_{S t}(d)=\frac{g \Delta \rho d^{2}}{18 \mu_{c}}
$$

$250>R e_{d}>1$ by the Allen formula:

$$
V_{A l}(d)=\sqrt{\left(\frac{g \Delta \rho}{\rho_{c}}\right)^{2} \cdot\left(\frac{\rho_{c}}{\mu_{c}}\right)} \cdot 0.24 d
$$

and when $R e_{d}>250$ by the Newton formula:

$$
V_{N}(d)=\sqrt{\frac{4 g \Delta \rho d}{3 \rho_{c}}},
$$

where $\rho_{\mathrm{c}}$ and $\rho_{d}$ - the density of the continuous medium, respectively, $\Delta \rho=\rho_{\mathrm{c}}-\rho_{d}$ - the difference of densities of phases of dispersed system, and if $\Delta \rho>0$ then the dispersed particle is deposited, but otherwise, floats, $g$ - the gravitational constant.

Relative motion mode of dispersed particles is characterized by the Reynolds number, in accordance with the expression $\operatorname{Re}_{d}=\rho_{c} V(d) \cdot d / \mu_{c}$.

In coarse dispersed systems of turbulent pulsations of the liquid is attached to the particle acceleration $a_{T}$, enabling them to resist the gravitational forces [20]. This is the main basic mechanism of droplets weighing. In the inertial interval at $\lambda_{0}<d$ on the basis of a dimensional analysis, the local acceleration of the fluid element with the size of a particle is written as $a_{T}=\left(e^{2} / d\right)^{1 / 3}$. Suppose that it is enough for weighing of the particles that force of gravity are balanced by inertial forces of a homogeneous turbulent flow. Then the condition for determining $d_{d e p}$ takes the following form:

$$
\varepsilon^{2 / 3}=C \cdot g \cdot \Delta \rho \cdot d_{d e p}^{1 / 3} / \rho_{c} .
$$

Using for energy dissipation in a turbulent flow through the pipeline with diameter $D$ the expression, the result is a model dependence of the inertial mechanism of weighing $U^{*}$ :

$$
\frac{U^{*}}{\sqrt{2 g D}} \cdot \sqrt{\frac{\rho_{c}}{\Delta \rho}}=C_{1} \lambda^{-1 / 3} \cdot\left(\frac{d_{d e p}}{D}\right)^{1 / 6},
$$

where $\lambda$ - the coefficient of hydraulic resistance. 
The critical value $U^{*}$ of average flow velocity for an accepted mechanism of weighing must increase monotonically with the increase of size $d_{d e p}$. However, it turned out that the dependence corresponds only to the initial portions of empirical curves (Rozentsvaig [20]). Subsequent increase of $d_{d e p}$ leads only initially to some reduction of the critical velocity. Then the value $U^{*}$ stabilizes and stops to depend considerably on the size of the dispersed particles. Such a complex non-linear dependency reflects the presence of other mechanisms of the particles interaction with turbulent flow along with the pulsation mechanism.

It is obvious that with the large particles diameter increase, the dynamic effect of turbulent flow on them is reduced in comparison with the force of gravity. Viscous interaction with the stream increases proportionately to the increase of the surface area. The tendency of increase the local viscous forces in a turbulent flow is formally possible to express with help of a corresponding decrease of the density difference of particles and the continuous medium of the emulsion $\Delta \rho$.

Let us consider the inclusion of the mechanism of the viscous interaction of particles and turbulent flow under the influence of gravity in an inertial mechanism. For this reason we introduce the effective density difference of the phases $\Delta \rho_{e f}$. Taking into account the dimensionless form of record it is represented with help of simple ratio of viscosity forces and gravitational forces with unknown exponential constant:

$$
\Delta \rho_{e f}=\Delta \rho \cdot\left(\frac{\mu_{c} \sqrt{2 \varepsilon / 15 v_{c}}}{\Delta \rho \cdot g \cdot d_{d e p}}\right)^{\beta}
$$

Constant $\beta$ is determined from the condition of independence of the diameter of the weighed droplets from the Froude number after substituting in the formula (13) expression $\Delta \rho_{\text {ef }}$ instead of $\Delta \rho$. The result is the value of $\beta=1 / 3$, with which the expression of model takes the form, which is consistent with experimental data:

$$
\frac{U^{*}}{\sqrt{2 g D}} \sqrt{\frac{\rho_{c}}{\Delta \rho}}=\text { const }
$$

with a precision up to the constant on the right side of the formula.

Particle deposition velocity in turbulent flow of the emulsion $U_{\text {turb }}(W, d)$ can be represented as:

$$
U_{t u r b}(W, d)=V_{d e p}(W, d)-S(W, d) \cdot \bar{v}(W),
$$

where $\bar{v}(W)$ represents the concentration dependence of the rate of pulsations [11]. The function $S(W, d)$ expresses the mechanisms of weighing and must be considered for specific tasks. However, if the size of particles, which are weighed in turbulent flow, is known then approximation of this function can be obtained under the condition:

$$
V_{d e p}\left(W, d_{d e p}\right)-S\left(W, d_{d e p}\right) \cdot \bar{v}(W)=0 .
$$

\subsection{Structure of turbulent flow of unstable liquid emulsions}

Interaction between dispersed phase droplets in liquid emulsions in turbulent regime increases the efficiency of heat exchange and mass transfer. But the concentration and the size of dispersed phase droplets change significantly the flow and hydrodynamic characteristics of two-phase flow (Balachandar and Eaton [21]). A significant factor is the structural form of the turbulent flow (Montoya [22]). Accounting a topology of the phases (droplets, bubbles, ligaments or any continuous structures) simplifies the modeling of turbulent multiphase systems greatly (Andreini et al. [23]).

Such turbulence models, as standard $k-\varepsilon$ model, RNG $k-\varepsilon$ model, the Reynolds stress model and large eddy simulation are well studied for homogeneous liquids (Joshi et al. [24]). However, when using turbulence models inherent in dispersed systems simplifying assumptions could describe accurately the influential aspects of the flow (Hakansson et al. [25]). Typical in this regard are the turbulent flows of liquid emulsions with nonequilibrium dispersed phase, capable for coalescing or breakup.

In particular, the nature of interaction of dispersed phase with a turbulent flow of the continuous medium of the emulsion depends on the ratio of droplets sizes and turbulent vortex. Dimensional characteristics of velocity fluctuations are determined by the local properties of turbulent flow of the emulsion. Turbulent mixing initiates coalescence of droplets of dispersed phase which can result in coalescence or fragmentation. As a result for implementation of those basic physical phenomena mechanisms there is a favorable current condition. These mechanisms specify the composition of the DPB-models taking into account mode and hydrodynamic parameters of the emulsion flow. But the turbulence structure in such multiphase systems, in turn, is defined with help of CFD- model. Therefore both of them need to coordinate their specifications, including relevant MBPP in both models.

With the sufficiently intense turbulent mixing of the emulsion the reverse effect of droplet size on the vortex motion of a continuous medium in a first approximation can be evaluated in the framework of the theory of locally isotropic turbulence. The redistribution of kinetic energy between the continuous medium and the dispersed phase is dynamic by nature only with close and smaller turbulent vortices. Otherwise the droplets are inside the vortices and the interaction of the phases of the emulsion takes a viscous character. When the droplets size is less than $\lambda_{0}$, the effect of the concentration of the dispersed phase on the hydrodynamic parameters is almost entirely limited to effective viscosity of the emulsion (Rosentsvaig and Pergushev [8]).

In coarse emulsions the diameter of droplets in which is $d>\lambda_{0}$, the interaction with a continuous environment is complicated. It does not reflect completely the usual exponential increase in effective viscosity with the increase of emulsion concentration. The effect of turbulent fluctuations damping by large droplets is 


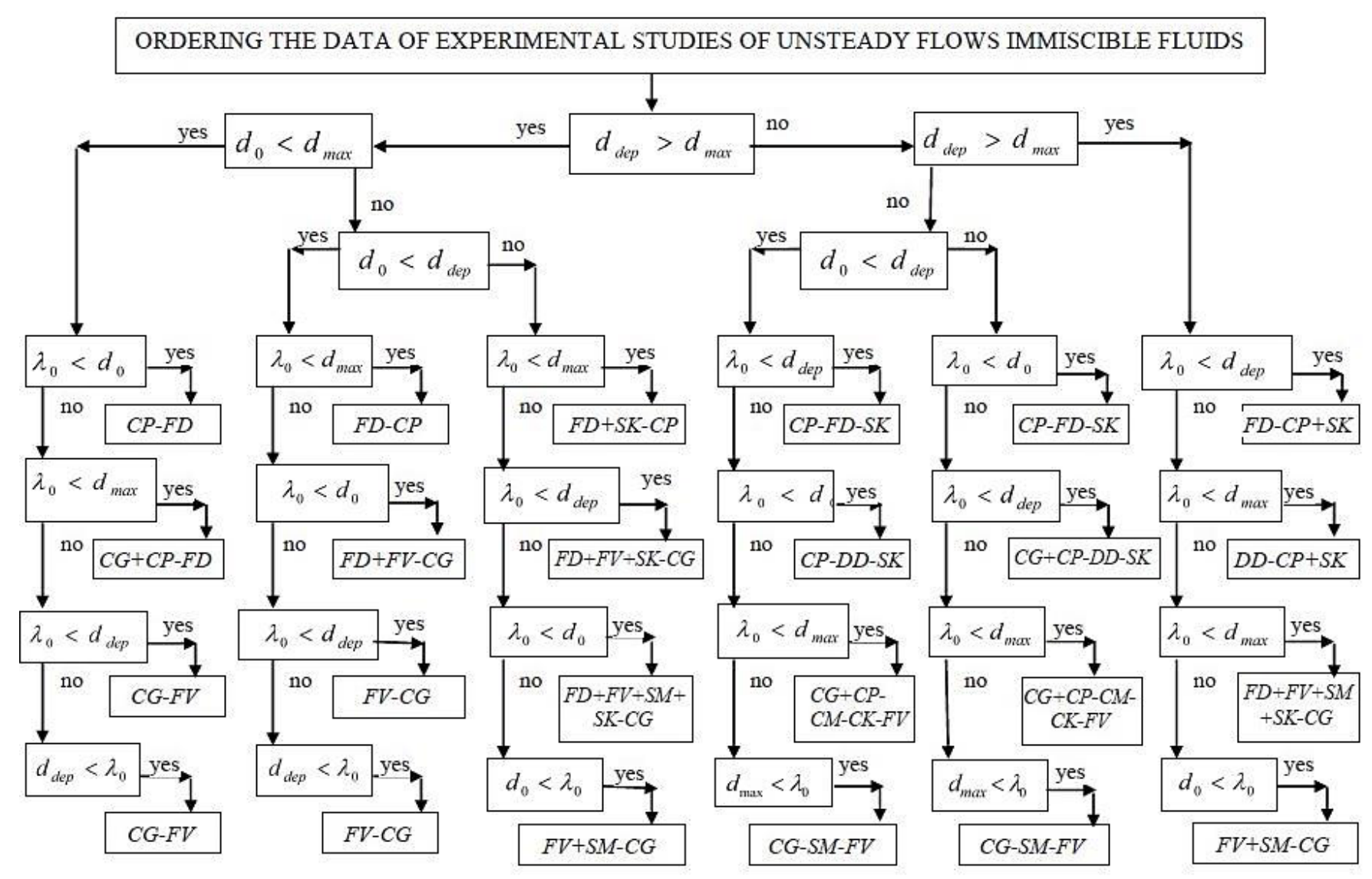

Fig. 3 Consistency of structures of experimental data and agreed CFD-DPB models

manifested indirectly by the weakening of concentration dependence of viscosity up to a near-linear growth (Rosentsvaig [26]).

Evaluation of the return influence on the turbulence characteristics of the continuous medium in size and concentration of dispersed droplets is required for the frequency calculation of the coalescence and fragmentation in unstable emulsions.

\section{Estimation of the parameters of the agreed models}

Reliability of statistical estimation parameters of complex phenomenological models is largely predetermined not only by the data of experimental measurements. They reflect the result of the implementation of cross-interrelated physical phenomena in the experimental setup. Therefore, the MBPP composition, included in the model, should correspond to the composition of basic physical phenomena, presented by the data of direct measurements under real conditions. In turn, this a priori provides an adequate interpretation of the results of the numerical analysis. This is especially important for matching them with the data of experimental studies, which in themselves do not always conform to the model concepts.

In particular, when boiling in liquid emulsions heat and mass transfer are associated with multifactor, fast-flowing processes, which are not always even possible to observe and measure in full scale (Zeigarnik [27]). Also the theoretical concepts that substantiate the model of transfer in unstable liquid emulsions are not unambiguous and are associated with severe restrictions. Methods of continuum mechanics cannot reflect all physical features of discrete dispersed systems. Universal abstract methods of analysis are also formal tools, the results of which require further interpretation.

The experimental measurements data for the model approximation interpretation must be received under conditions where the same MBPP model composition is realized. For this purpose the selection of criteria presented in Fig. 3, supported in accordance with the experimental data and the composition of specified models is necessary. Evaluations of the model constants in this case take the general character and retain their numerical values at some other MBPP combinations and conditions of the external and internal environment. Such an approach allows highlighting the role of individual mechanisms in the formation of complex physical processes (Galbraith [28]).

In addition, estimation of parameters must be performed using both agreed CFD and DPB models. Otherwise, the most advanced statistical procedure leads to unreliable and unrealistic evaluation of the model parameters. The data of experimental studies, systematic and orderly in Fig. 3 in accordance with the hierarchical structure of the model, turn into a coherent database. When performing parametric identification using the database, which structure corresponds to the structure of the model, the reliability of the estimates of the constants of the model relations is provided. 


\section{Conclusions}

Physical transfer processes in liquid emulsions are naturally interrelated with boundary conditions and hydrodynamic processes of momentum transfer. A character of relationship predetermines the peculiarities of the specifications of composition and structure of models, because in commonality of the basic elements - mechanisms of the elementary physical phenomena is evident.

When using both hydrodynamic CFD and population DPB models for numerical analysis of a turbulent flow of unstable emulsions, it is necessary to adjust the composition and interrelationships of physical phenomena in the dispersed phase. Fragmentation, coalescence and sedimentation of the droplets of the dispersed phase flow are ambiguous, depending on hydrodynamic and boundary conditions of emulsions. In turn, the turbulence characteristics have the opposite effect of the spectrum of size distributions and concentrations of dispersed droplets. For joint CFD and DPB models an adjustment of model representations of the interconnected transfer processes is needed.

Using the $d_{\max }, d_{d e p}$ and $\lambda_{0}$ criteria the detailization of the complex physical processes composition in turbulent flows of unstable emulsions is done. The mechanisms of the basic physical phenomena (MBPP) are highlighted, which determine not only the composition but also the physical interpretation of the simulation results. An essential circumstance of this approach of modeling is construction of space (of the full set) of basic physical phenomena. In this space a set of common elements is created for the adjustment of specifications of model representations.

With help of destabilization criteria a priori empirical information is generalized, which is not amenable to a complete formalization in the case of the interconnected transfer processes. They needed to establish the conditions of applicability of particular MBPP structures for providing the adequacy of the phenomenological models in the form of linear relationships.

Model concepts of complex hydrodynamic behavior of dispersed systems are important for the analysis of mechanisms of mass transfer, heat transfer and boiling in emulsions. With help of these models will be highlighted the detail of conditions of mass transfer and heat for each subject area (Rozentsvaig and Strashinskii [29]). In turn, their mechanisms are totally dependent on the dispersion of droplets and from the nature of the processes of their breakup/coalescence, as well as from the hydrodynamics behavior of the emulsion. Ultimately, the structural approach allows us to interpret the more complex processes of heat transfer in unstable emulsions.

\section{References}

[1] Xing, C., Wang, T., Wang, J. "Experimental study and numerical simulation with a coupled CFD-PBM model of the effect of liquid viscosity in a bubble column." Chemical Engineering Science. 95, pp. 313-322. 2013. https://doi.org/10.1016/j.ces.2013.03.022

[2] Gao, Z., Li, D., Buffo, A., Podgórska, W., Marchisio, D. L. "Simulation of droplet breakage in turbulent liquid-liquid dispersions with CFDPBM: Comparison of breakage kernels." Chemical Engineering Science. 142, pp. 277-278. 2016.

https://doi.org/10.1016/j.ces.2015.11.040

[3] Ramkrishna, D. "Population balances. Theory and applications to particulate systems in engineering." Academic Press, New York, NY. 2000.

[4] Ramkrishna, D., Mahoney, A. W. "Population balance modeling. Promise for the future." Chemical Engineering Science. 57(4), pp. 595-606. 2002. https://doi.org/10.1016/S0009-2509(01)00386-4

[5] Rozentsvaig, A. K., Strashinskii, Ch. "Mechanisms of boiling of an emulsion with a low-boiling disperse phase in a turbulent flow of a homogeneous emulsion." Journal of Engineering Physics and Thermophysics. 83(3), pp. 486-495. 2010. https://doi.org/10.1007/s10891-010-0369-4

[6] Yeoh, G. H., Tu, J. Y. "Two-fluid and population balance models for subcooled boiling flow." Applied Mathematical Modelling. 30, pp. 1370 1391. 2006.

https://doi.org/10.1016/j.apm.2006.03.010

[7] Abidin, M., Raman, A., Nor, M. "Mean drop size correlations and population balance models for liquid-liquid dispersion." AIChE Journal. 61(4), pp. 1129-1145. https://doi.org/10.1002/aic.14751

[8] Rozentsvaig, A. K., Pergushev, L.P. "Coalescence of concentrated finedisperse emulsions during turbulent stirring." Journal of Engineering Physics. 40(6), pp. 608-612. 1981. https://doi.org/10.1007/BF00825447

[9] Rozentsvaig, A. K. "Breakup of droplets in turbulent shear flow of dilute liquid-liquid dispersions." Journal of Applied Mechanics and Technical Physics. 22(6), pp. 797-802. 1981. https://doi.org/10.1007/BF00906109

[10] Rosentsvaig, A. K. "Coalescence of coarsely dispersed concentrated emulsions with turbulent agitation." Journal of Engineering Physics. 42(1), pp. 19-24. 1982.

https://doi.org/10.1007/BF00824984

[11] Rozentsvaig, A. K. "Turbulent pipe flow of concentrated emulsions with a non-equilibrium disperse phase." Journal of Engineering Physics. 42(3), pp. 254-259. 1982. https://doi.org/10.1007/BF00827741

[12] Rozentsvaig, A. K., Strashinskii, C. S. "Identification of models of transfer processes in complex disperse systems." Applied Mathematical Sciences. 10(24), pp. 1151-1161. 2016. https://doi.org/10.12988/ams.2016.6137

[13] Rozentsvaig, A. K. "Numerical analysis of equilibrium droplet spectrum formation in turbulent transverse flow of polydispersed concentrated emulsions." Journal of Applied Chemistry of the USSR. 61, pp. 2450-2455. 1988.

[14] Zhang, Y., Jia, M., Liu, H., Xie, M. "Development of an improved liquid film model or spray/wall interaction under engine-relevant conditions." International Journal of Multiphase Flow. 79, pp. 74-87. 2016. https://doi.org/10.1016/j.ijmultiphaseflow.2015.10.002

[15] Kolmogorov, A. N. "On the breakage of drops in a turbulent flow." Doklady Akademii Nauk SSSR. 66. pp. 825-828. 1949. 
[16] Taylor, G. I. "The formation of emulsions in definable fields of flow." Proceedings of the Royal Society A: Mathematical, Physical \& Engineering Sciences. 146, pp. 501-523. 1934.

https://doi.org/10.1098/rspa.1934.0169

[17] Slecher, C. Jr. "Maximum stable drop size in turbulent flow." AIChE Journal. 8, pp. 471-477. 1962. https://doi.org/10.1002/aic.690080410

[18] Sarimeseli, A., Kelbaliyev, G. "Deposition of dispersed particles in isotropic turbulent flow." Journal of Dispersion Science and Technology. 29(2), pp. 307-312. 2008. https://doi.org/10.1080/01932690701688839

[19] Ryazhskikh, A. V., Boger, A. A., Slyusarev, M. I., Ryazhskikha, V. I. "Convective-diffusion model of transfer of a sedimenting low-concentration polydisperse suspension of stokesian particles in a plane channel. Part 1." Journal of Engineering Physics and Thermophysics. 89(1), pp. 10-18. 2016.

https://doi.org/10.1007/s10891-016-1348-1

[20] Rozentsvaig, A. K. "Structure analysis of mechanism of weighing of particles in a turbulent flow of liquid disperse systems." Izvestiâ Vysših Učebnyh Zavedenij. Problemy Energetiki. No. 9-10, pp. 19-34. 2003. (in Russian)

[21] Balachandar, S., Eaton, J. "Turbulent dispersed multiphase flow." Annual Review of Fluid Mechanics. 42(1), pp. 111-133. 2010. https://doi.org/10.1146/annurev.fluid.010908.165243

[22] Montoya, G., Lucas, D., Baglietto, E., Liao, Y. "A review on mechanisms and models for the churn - turbulent flow regime." Chemical Engineering Science. 141(2), pp. 86-103. 2016. https://doi.org/10.1016/j.ces.2015.09.011

[23] Andreini, A., Bianchini, C., Puggelli, S., Demoulin, F. X. "Development of a turbulent liquid flux model for Eulerian-Eulerian multiphase flow simulations." International Journal of Multiphase Flow. 81, pp. 88-103. 2016. https://doi.org/10.1016/j.ijmultiphaseflow.2016.02.003
[24] Joshi, J., Nere, N., Rane, C., Murthy B., Mathpati C., Patwardhan, A., Ranade, V. "CFD simulation of stirred tanks: Comparison of turbulence models. Part I: Radial flow impellers." The Canadian Journal of Chemical Engineering. 89(1), pp. 23-82. 2011.

https://doi.org/10.1002/cjce.20446

[25] Hakansson, A., Fuchs, L., Innings, F., Revstedt, J., Tragardha, Ch., Bergenstahl, B. "Experimental validation of $k-\varepsilon$ RANS-CFD on a highpressure homogenizer valve." Chemical Engineering Science. 71, pp. 264-273. 2012.

https://doi.org/10.1016/j.ces.2011.12.039

[26] Rozentsvaig, A. K. "Mechanism of the comminution of drops during the mixing of dilute emulsions with turbine-driven agitators." Journal of Applied Chemistry of the USSR. 58, pp. 1191-1198. 1985.

https://doi.org/10.13140/RG.2.1.4152.1527

[27] Zeigarnik, Yu., Platonov, D, Khodakov, K., Shekhter, Yu. "Visualization of boiling of subcooled water." High Temperature. 49(4), pp. 566-570. 2011. https://doi.org/10.1134/S0018151X11020179

[28] Galbraith, S. C., Schneider, P. A., Flood, A. E. "Model-driven experimental evaluation of struvite nucleation, growth and aggregation kinetics." Water Research. 56(6), pp. 122-132. 2014. https://doi.org/10.1016/j.watres.2014.03.002

[30] Rozentsvaig, A. K., Strashinskii, C. S. "Modeling of heat transfer conditions in cooling lubricant emulsions with low-boiling continuous media in narrow gaps." International Journal of Heat and Mass Transfer. 102, pp. 550-560. 2016.

https://doi.org/10.1016/j.ijheatmasstransfer.2016.06.072 